\title{
Diffractive Higgs bosons and prompt photons at hadron colliders
}

\author{
R. Enberg, ${ }^{1}$ G. Ingelman, ${ }^{1,2}$ and N. Tîmneanu ${ }^{1}$ \\ ${ }^{1}$ High Energy Physics, Uppsala University, Box 535, S-75121 Uppsala, Sweden \\ ${ }^{2}$ Deutsches Elektronen-Synchrotron DESY, Notkestrasse 85, D-22603 Hamburg, Germany
}

\begin{abstract}
Models for soft color interactions have been successful in describing and predicting diffractive hard scattering processes in $e p$ collisions at DESY HERA and $p \bar{p}$ at the Fermilab Tevatron. Here we present new comparisons of the model to recent diffractive dijet data, also showing good agreement. The topical issue of diffractive Higgs production at the Tevatron and LHC hadron colliders is further investigated. For $H \rightarrow \gamma \gamma$ the irreducible background of prompt photon pairs from $q \bar{q} \rightarrow \gamma \gamma$ and $g g \rightarrow \gamma \gamma$ is always dominating, implying that higher branching ratio decay modes of the Higgs have to be used. However, such prompt photons can be used to test the basic prediction for Higgs production since $g g \rightarrow \gamma \gamma$ involves a quark loop diagram similar to $g g \rightarrow H$.
\end{abstract}

PACS numbers: 14.80.Bn, 12.38.Lg, 13.85.Rm

\section{INTRODUCTION}

There has recently been much interest 11, 2, 3, 4, 5, 6] in whether the Higgs boson could be easier observed, or even discovered, in diffractive scattering events at hadron colliders, viz. $p \bar{p}$ at the Fermilab Tevatron or $p p$ at CERN's future Large Hadron Collider (LHC). The underlying idea is that the lower hadronic activity in such events with large rapidity gaps should improve the possibility to reconstruct the Higgs from its decay products. One crucial issue is whether the cross section for diffractive Higgs production is large enough to give an observable rate. Theoretical predictions based on different models vary by orders of magnitude and it is therefore important to check and constrain these models in various ways.

Another crucial issue is which decay mode of the Higgs that is considered. Although $H \rightarrow \gamma \gamma$ (Fig. 1 b) has a small branching ratio, its very clear experimental signature with two high- $p_{\perp}$ photons makes it interesting and often considered for a Higgs of intermediate mass.

In this note we will address both these crucial issues. Our prediction [2] for the diffractive Higgs cross section is given additional credibility by further experimental checks supporting our soft color interaction (SCI) model. The $H \rightarrow \gamma \gamma$ channel is further considered by investigating the background from prompt $\gamma \gamma$ production. The process $g g \rightarrow \gamma \gamma$ is similar to the dominating Higgs production process $g g \rightarrow H$, both involving gluon-gluon fusion into a quark loop (Fig. 11). The experimental observation of prompt photon pairs in rapidity gap events can therefore be used to test the models for diffractive Higgs production. We therefore give predictions on such prompt photon events based on our model.

\section{DIFFRACTIVE HARD SCATTERING IN THE SCI MODEL}

The soft color interaction (SCI) model [7] was developed in an attempt to better understand non-

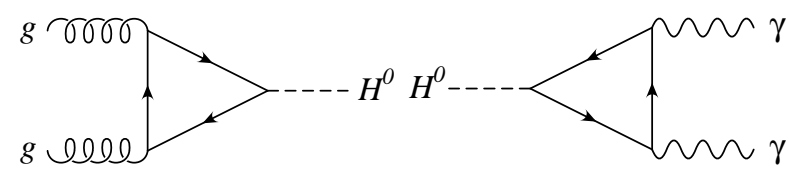

(a)

(b)

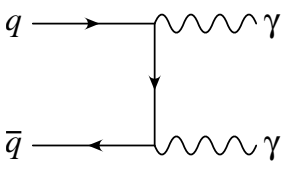

(c)

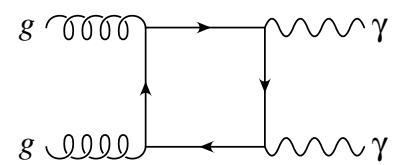

(d)
FIG. 1: Higgs production in (a) gluon-gluon fusion via a quark loop and decay (b) via a quark or $W$ loop into a photon pair. Prompt photon pair production in (c) leading order quarkantiquark annihilation and in (d) higher order gluon-gluon fusion via a quark loop.

perturbative QCD dynamics and provide a unified description of all final states. The basic assumption is that soft color exchanges give variations in the topology of the confining color string-fields which then hadronize into different final states, e.g. with and without rapidity gaps or leading protons. Also other kinds of experimental results are described in a very economical way with only one new parameter. Particularly noteworthy is the turning of a $c \bar{c}$ pair from a color octet state into a singlet state producing charmonium [8] in good agreement with observed rates.

The SCI model 77 is implemented in the Lund Monte Carlo programs LEPTO [9] for deep inelastic scattering and PYтнia [10] for hadron-hadron collisions. The hard parton level interactions are given by standard perturbative matrix elements and parton showers, which are not altered by the softer non-perturbative effects. The SCI model then applies an explicit mechanism where coloranticolor (corresponding to non-perturbative gluons) can be exchanged between the emerging partons and hadron remnants. The probability for such an exchange cannot be calculated and is therefore taken to be a constant given by a phenomenological parameter $P$. These color 


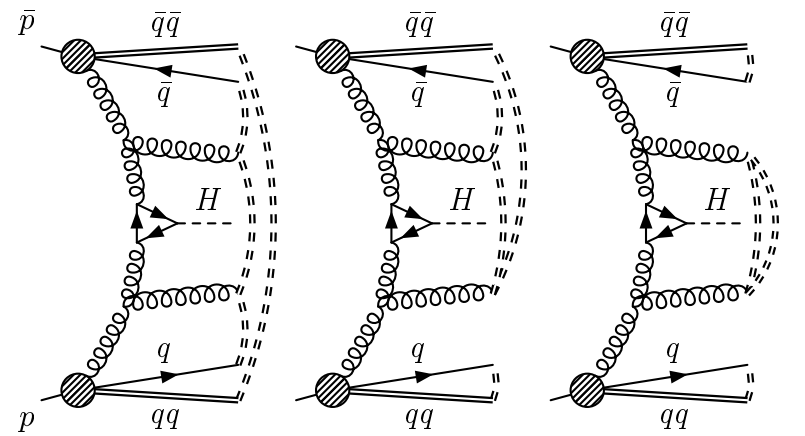

FIG. 2: Higgs production in $p \bar{p}$ collisions with string topologies (double-dashed lines) before and after soft color interactions in the SCI model, resulting in events with one or two rapidity gaps (leading protons).

exchanges modify the color connections between the partons and thereby the color string-field topology, as illustrated in Fig. 2. Standard Lund model hadronization [1] of the string fields then leads to different final states, with gaps in rapidity regions where no string was present. Following the normal factorization theorem, these soft processes do not affect the cross section for the hard scattering process, but only the distribution of hadrons in the final state.

A variation is provided by the generalized area law (GAL) model [12], which is formulated in terms of interactions between the strings and not the partons. The probability for two strings to interact via a soft color exchange is obtained from the area law in the Lund string model resulting in a dynamically varying interaction probability. The results of this model are in practice very close to those of the SCI model and we will therefore in this short note only consider the SCI model.

Since the SCI model is implemented in Monte Carlo programs which generate complete events with final state particles, one can adopt an experimental approach to classify events depending on the final state, i.e. diffractive or non-diffractive depending on the presence of rapidity gaps or leading (anti)protons. The value of the single parameter $P$, regulating the amount of soft color exchanges, is determined from the rate of diffractive deep inelastic events observed in $e p$ at HERA. The predictive power of the model lies in the fact that applying it, with the same parameter value $(P=0.5)$, to $p \bar{p}$ gives a good description of all diffractive hard scattering processes observed at the Tevatron, as summarized in Table 1 .

The PYTHIA Monte Carlo has here been employed for different hard subprocesses producing jets, $W, Z, c \bar{c}$ or $b \bar{b}$. The overall cross sections are obtained as usual by folding the subprocess cross sections with the parton density distributions (we have used CTEQ [13 as detailed in [14]). The SCI model is then applied before hadronization is performed and the events classified. Single diffractive (SD) events are selected using one of two criteria: (1) a leading (anti)proton with $x_{F}>0.9$ or (2) a rapid-
TABLE I: Ratios diffractive/inclusive for hard scattering processes in $p \bar{p}$ collisions $(\sqrt{s}=1800 \mathrm{GeV})$ at the Tevatron, showing experimental results from CDF 15, 16 and D $\varnothing 17$ compared to the soft color interaction (SCI) model.

\begin{tabular}{|c|c|c|}
\hline \multirow[b]{2}{*}{ Observable } & \multicolumn{2}{|c|}{ Ratio $[\%]$} \\
\hline & Observed & SCI model \\
\hline$W-$ gap & $\mathrm{CDF} 1.15 \pm 0.55$ & 1.2 \\
\hline$Z$ - gap & $\mathrm{D} \varnothing \quad 1.44_{-0.54}^{+0.62}$ & $1.0^{a}$ \\
\hline$b \bar{b}-$ gap & CDF $0.62 \pm 0.25$ & 0.7 \\
\hline$J / \psi-$ gap & $\mathrm{CDF} 1.45 \pm 0.25$ & $1.4^{a}$ \\
\hline$j j-$ gap & CDF $0.75 \pm 0.10$ & 0.7 \\
\hline$j j-$ gap & $\mathrm{D} \varnothing \quad 0.65 \pm 0.04$ & 0.7 \\
\hline gap - $j j-$ gap $^{b}$ & CDF $0.26 \pm 0.06$ & 0.2 \\
\hline $\bar{p}-j j-$ gap $^{b}$ & CDF $0.80 \pm 0.26$ & 0.5 \\
\hline \multicolumn{3}{|c|}{${ }^{a}$ Prediction of model } \\
\hline${ }^{b}$ Ratio DPE/S & & \\
\hline
\end{tabular}

ity gap in the forward or backward region, for example $2.4<|\eta|<5.9$ as used by the CDF collaboration. Applying the conditions in both hemispheres results in events with two rapidity gaps, one on each side of the central hard scattering system. These are conventionally labeled DPE after their interpretation as double pomeron exchange in the Regge framework, but we prefer to use this established acronym to denote 'Double leading Proton Events' independently of interpretations in terms of any specific model.

For a detailed comparison of the SCI model with results on diffractive hard scattering at the Tevatron see [14. Here we want to point out that some of our results were predictions that have recently been verified by new experimental data, as indicated in Table 1 .

A process which has drawn much attention recently is the central production of dijets in DPE events, which has been observed at the Tevatron 15. The SCI model describes well the cross section and the ratio of these events to single diffractive dijet events (as shown in Table i]), which indicates the breakdown of diffractive factorization [14, 15]. Also more exclusive quantities, such as transverse energy of the jets or their rapidity distribution are well described 114].

In Fig. 3 we show that another new measurement is well described by the SCI model. This dijet mass fraction $\mathcal{R}_{j j}$ is an interesting observable that shows how large fraction of the available energy in the central system of DPE events that goes into the hard scattering system and produces the two jets in the final state. Events with $\mathcal{R}_{j j}$ close to unity would correspond to an exclusive jet production process and the data limits such a contribution to $\sigma \lesssim 3.7 \mathrm{nb}[15]$ just above a model prediction of $\sim 1 \mathrm{nb}$ [3]. The observed dijet mass fraction is instead concentrated at smaller values, leaving a substantial energy fraction in the remaining central DPE system, in accordance with the jet production mechanism in the SCI model. 


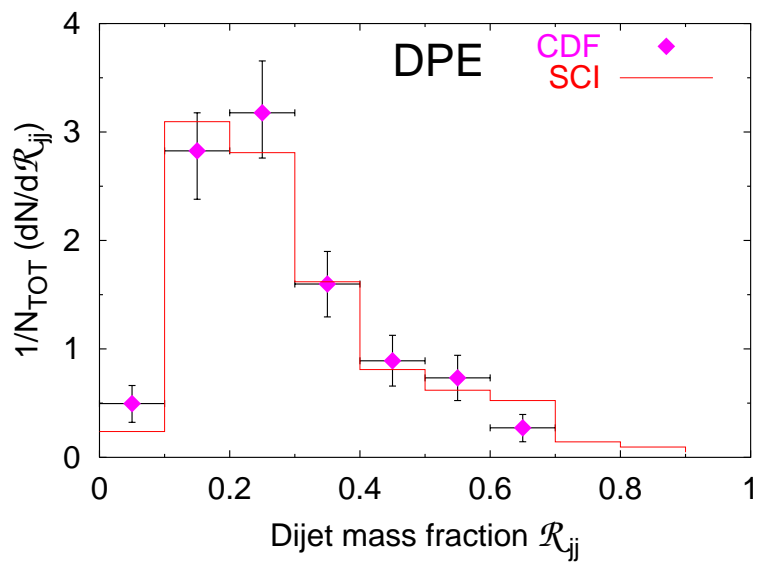

FIG. 3: Distribution of the dijet mass fraction, i.e. ratio $\mathcal{R}_{j j}$ of the invariant mass of the dijet system to the invariant mass of the central hadronic system in DPE events. CDF data 15 compared to the SCI model.

Turning now to Higgs production, we select those hard subprocesses in PYTHIA which produce a Higgs boson. The dominant one is $g g \rightarrow H$ via a quark loop as illustrated in Fig. 17a, which accounts for $50 \%$ and $70 \%$ of the cross section (for $115<m_{H}<200 \mathrm{GeV}$ ) at the Tevatron and LHC, respectively. Other production channels $\left(q_{i} \bar{q}_{i} \rightarrow H, q_{i} \bar{q}_{i} \rightarrow Z H, q_{i} \bar{q}_{j} \rightarrow W H, q_{i} q_{j} \rightarrow q_{k} q_{l} H\right.$ and $\left.g g \rightarrow q_{k} \bar{q}_{k} H\right)$ contribute, depending both on the Higgs mass and the center of mass energy. Our results in the following are based on these leading order processes, but we note that higher order corrections could give an increase of the Higgs production cross section with an effective $K$-factor of order two 18 .

Applying the same SCI model on the resulting partonic state gives rise to different color string topologies, resulting in different final states after the standard Lund model [1] has been applied for hadronization (Fig. 2). Classifying events as above, depending on rapidity gaps or leading protons, we obtained the results for diffractive Higgs production as shown in detail in [2]. We summarize the main results in terms of overall cross sections in Table III.

At the Tevatron, the cross section for Higgs in DPE events, which would have the least disturbing underlying hadronic activity, is too small to give any produced events. Higgs in single diffractive events are produced, but in small numbers such that only the decay mode $H \rightarrow b \bar{b}$ with the largest branching ratio will give any events to search for.

At LHC, the high energy and luminosity facilitates a study of single diffractive Higgs production, where also the striking $H \rightarrow \gamma \gamma$ decay should be observed. Also a few DPE Higgs events may be observed, but these events will not be as clean as naively expected. The available energy is here enough to produce the Higgs and the leading protons as well as an underlying event that will popu-
TABLE II: Cross sections and numbers (\#) of events at the Tevatron and LHC for Higgs in single diffractive (SD) and DPE events, defined by leading protons or rapidity gaps, obtained from the soft color interaction model (SCI).

\begin{tabular}{cccc}
\hline \hline \multirow{2}{*}{$m_{H}=115 \mathrm{GeV}$} & \multicolumn{2}{c}{ Tevatron } & LHC \\
& $\sqrt{s}=1.96 \mathrm{TeV}$ & $\sqrt{s}=14 \mathrm{TeV}$ \\
& $\mathcal{L}=20 \mathrm{fb}^{-1}$ & $\mathcal{L}=30 \mathrm{fb}^{-1}$ \\
\hline & $\sigma[\mathrm{fb}]$ Higgs-total & 600 & 27000 \\
\hline $\mathrm{SD}$ & $\sigma[\mathrm{fb}]$ leading-p & 1.2 & 190 \\
& $\sigma[\mathrm{fb}]$ gap & 2.4 & 27 \\
\# H + leading-p & 24 & 5700 \\
$\hookrightarrow$ \# H $\rightarrow \gamma \gamma$ & 0.05 & 13 \\
\hline DPE $\sigma[\mathrm{fb}]$ leading-p's & $1.2 \cdot 10^{-4}$ & 0.19 \\
$\sigma[\mathrm{fb}]$ gaps & $2.4 \cdot 10^{-3}$ & $2.7 \cdot 10^{-4}$ \\
$\# \mathrm{H}+$ leading-p's & 0.0024 & 6 \\
\hline \hline
\end{tabular}

late forward detector rapidity regions with particles [2]. This causes a much lower diffractive cross section when requiring a gap instead of a leading proton at LHC.

\section{III. $H \rightarrow \gamma \gamma$ VERSUS PROMPT $\gamma \gamma$}

The $H \rightarrow \gamma \gamma$ decay mode is of experimental interest due to its clear signature with two photons of high $p_{\perp}$ (up to $m_{H} / 2$ ). Its branching ratio is, however, quite low since it proceeds via a higher order loop diagram (Fig. 1 $\mathrm{lb}$ ) and is only of interest for an intermediate mass Higgs, below the thresholds for decaying into $W^{+} W^{-}$ and $Z^{0} Z^{0}$. As the Higgs mass increases from $m_{H}=115$ $\mathrm{GeV}$ to $m_{H}=160 \mathrm{GeV}$, the branching ratio decreases from about $2 \times 10^{-3}$ to about $6 \times 10^{-4}[19]$. Therefore, this decay mode gives too low rates to be observable in diffractive interactions (Table II), except for a handful $H \rightarrow \gamma \gamma$ in single diffraction at LHC.

Concerning the backgrounds to this Higgs signal, the requirement of the two high- $p_{\perp}$ photons to be essentially back-to-back in the transverse plane and isolated, removes the major backgrounds of photons as decay products in jets. The serious, irreducible background is given by the production of a pair of prompt high- $p_{\perp}$ photons from the hard processes $q \bar{q} \rightarrow \gamma \gamma$ and $g g \rightarrow \gamma \gamma$ (Fig. 11 cd). As above, we simulate these processes with PYTHIA including the SCI model and obtain the inclusive cross sections, as well as the single diffractive and DPE ones shown in Fig. 1. The Tevatron cross sections for diffractive Higgs and prompt photons with high mass are very suppressed due to kinematics at the edge of phase space, while at the LHC, this kinematic suppression is much less pronounced.

As can be seen, the irreducible prompt $\gamma \gamma$ background is larger, in particular at the Tevatron, than the cross section times the branching ratio for $H \rightarrow \gamma \gamma$. This applies to inclusive, SD and DPE events when considering a bin width of only $\sim 1 \mathrm{GeV}$, corresponding to the width 

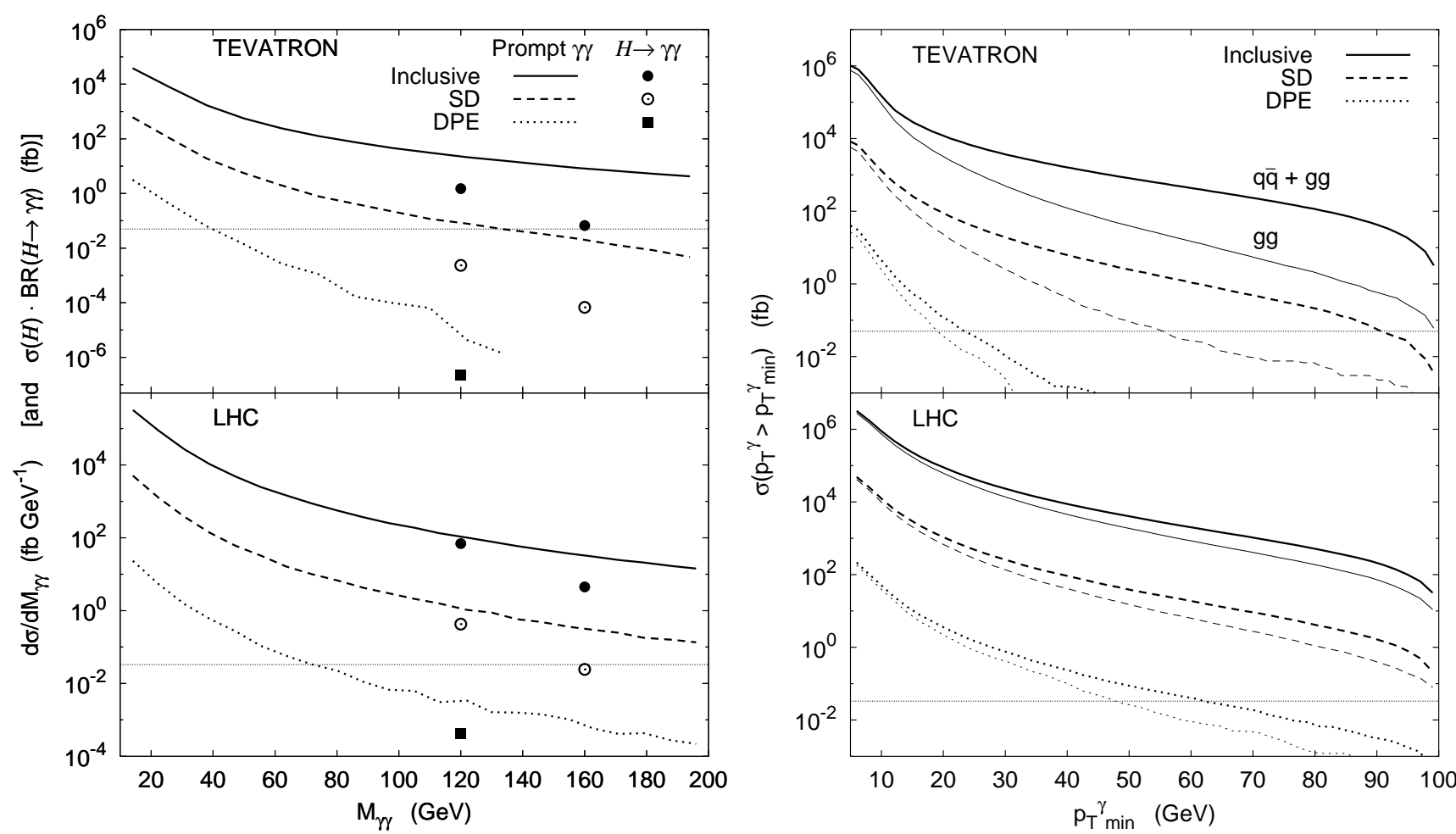

FIG. 4: Cross sections at the Tevatron and LHC for the production of prompt $\gamma \gamma$ (with $\left|\eta_{\gamma}\right|<2$ ) versus (a) their invariant mass $M_{\gamma \gamma}$ and (b) integrated from a minimum transverse momentum $p_{\perp \min }^{\gamma}$. Curves are predictions from the soft color interaction model; inclusive, single diffractive and DPE events and in (b) showing separately the contribution from the $g g \rightarrow \gamma \gamma$ process (thin lines). For comparison, $\sigma(H) \cdot \mathrm{BR}(\mathrm{H} \rightarrow \gamma \gamma)$ is shown in (a) for $m_{H}=120 \mathrm{GeV}\left(\mathrm{BR}=2.2 \times 10^{-3}\right)$ and $m_{H}=160 \mathrm{GeV}$ $\left(\mathrm{BR}=5.5 \times 10^{-4}\right)$. The horizontal lines show the cross-section for obtaining one event with the planned integrated luminosities of 20 and $30 \mathrm{fb}^{-1}$ at the Tevatron and LHC, respectively.

of a Higgs with $m_{H}=160 \mathrm{GeV}$ and a detector with good energy resolution. For a Higgs of lower mass the width is much smaller, e.g. $\sim 10 \mathrm{MeV}$ at $m_{H}=120 \mathrm{GeV}$, such that the $H \rightarrow \gamma \gamma$ signal could in priciple stand out from the background in $10 \mathrm{MeV}$ bins obtained from a detector with extremely high energy resolution. To be realistic, however, we must unfortunately conclude that $\gamma \gamma$ is not a straightforward signal for observing the Higgs boson.

On the other hand, the prompt $\gamma \gamma$ production has a large enough cross section to be investigated in diffractive events. At the Tevatron, observable rates are predicted for SD events with $p_{\perp}^{\gamma}$ up to $\sim 75 \mathrm{GeV}$, so the model can be tested against data even up to scales of order $m_{H} / 2$, but for DPE, $p_{\perp}^{\gamma}$ is only observable up to $15-20 \mathrm{GeV}$. For large $p_{\perp}^{\gamma}$ the leading order subprocess $q \bar{q} \rightarrow \gamma \gamma$ dominates. The higher order process $g g \rightarrow \gamma \gamma$ dominates, however, at lower $p_{\perp}^{\gamma}$ where the momentum fraction $x$ of the initial partons can be smaller and the large gluon density of the proton increases the cross section. Therefore, in order to test the model for a similar gluon-gluon fusion process with a quark loop as in Higgs production, one should consider $p_{\perp}^{\gamma}$ up to $\sim 15 \mathrm{GeV}$ for SD and up to $\sim 20 \mathrm{GeV}$ for DPE (Fig. 四) .

Similarly at the LHC, prompt $\gamma \gamma$ gives observable rates for SD for $p_{\perp}^{\gamma}$ up to $\sim 100 \mathrm{GeV}$ and for DPE up to $\sim 50$ $\mathrm{GeV}$, i.e. up to the scale of $m_{H} / 2$. The process $g g \rightarrow \gamma \gamma$ dominates for values of $p_{\perp}^{\gamma}$ below $\sim 60 \mathrm{GeV}$ for $\mathrm{SD}$ and below $\sim 40 \mathrm{GeV}$ for DPE.

Thus, the basic mechanism for producing rapidity gap events with a high mass state from $g g$ fusion via a quark loop can be tested already at the Tevatron and further investigated at the LHC.

\section{CONCLUDING DISCUSSION}

Diffractive Higgs production is currently considered with great interest for forthcoming investigations at the Tevatron and LHC. Different theoretical models predict quite different magnitudes of the cross section, as dis-

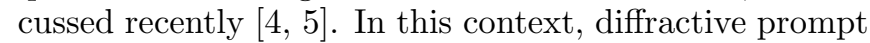
$\gamma \gamma$ production can be used as a further testing ground for the models. In particular, the contributing subprocess $g g \rightarrow \gamma \gamma$ involves a similar gluon-gluon fusion process via a quark loop as in Higgs production.

Although models based on pomeron exchange can be made to fit diffractive hard scattering data at both HERA and the Tevatron, they have conceptual and theoreti- 
cal problems as discussed in [14, 20]. In particular, it seems improper to regard the pomeron as 'emitted' from the proton and having QCD evolution as a separate entity. This problem is avoided in the soft color interaction model which, although not having a firm theoretical basis, can be seen as an effective model for final state interactions as discussed theoretically in [20]. This SCI model has, furthermore, strong phenomenological support since it has been very successful in describing and predicting diffractive hard scattering processes both in $e p$ collisions at HERA and $p \bar{p}$ at the Tevatron [14]. It should be emphasized that the SCI model describes well the production of dijets in DPE events - a process which has similar dynamics to DPE Higgs and has been advocated [5] as a testing ground for different models aiming at describing diffractive Higgs.

All this gives a high credibility to our predictions for diffractive Higgs [2] and prompt $\gamma \gamma$ presented in this note.

Based on the SCI model, we find that the rate of diffractive Higgs events at the Tevatron will be too low to be useful. Some single diffractive Higgs events should be produced, but they can only be accessed through the $H \rightarrow b \bar{b}$ decay channel which does not provide a clear signal in view of the large QCD background and non-trivial experimental $b \bar{b}$ reconstruction. The Higgs must, therefore, be primarily searched for in inclusive events with their more complex underlying event. The situation is quite different at LHC. Here single diffractive Higgs production can be investigated, including a few events with the striking $H \rightarrow \gamma \gamma$ decay mode, and a few DPE events with a Higgs should also be produced. Diffractive events are, however, not so clean at the LHC since the large available energy produces underlying event activity extending to larger forward rapidities making observable gaps less abundant. Therefore, a much larger sample of diffractive Higgs events would be obtained by very forward 'Roman pot'-type proton tagging detectors.

We furthermore find that the $H \rightarrow \gamma \gamma$ signal in all cases has a smaller cross section than the irreducible background of prompt $\gamma \gamma$ production. As discussed above, the latter process is interesting in its own right and our model gives observably large cross sections both at the Tevatron and LHC, such that prompt $\gamma \gamma$ event samples should be obtained inclusively as well as in single diffraction and DPE. A one to two orders of magnitude larger cross section for prompt $\gamma \gamma$ in DPE was predicted in [6] based on a pomeron model, but the uncertainty was considered large due to the poorly constrained Reggeon exchanges. Thus, the investigation of diffractive prompt $\gamma \gamma$ production at the Tevatron is essential to test the models for diffractive hard scattering and obtain safer predictions for diffractive Higgs production at the LHC.
[1] A. Bialas, P.V. Landshoff, Phys. Lett. B 256, 540 (1991); H.J. Lu and J. Milana, Phys. Rev. D 51, 6107 (1995); D. Graudenz, G. Veneziano, Phys. Lett. B365, 302 (1996); J.R. Cudell and O.F. Hernandez, Nucl. Phys. B 471, 471 (1996); M. Heyssler, Z. Kunszt and W. J. Stirling, Phys. Lett. B 406, 95 (1997); M. Boonekamp, R. Peschanski and C. Royon, Phys. Rev. Lett. 87, 251806 (2001); M. Boonekamp, A. De Roeck, R. Peschanski and C. Royon, Acta Phys. Polon. B 33, 3485 (2002). A. De Roeck, V. A. Khoze, A. D. Martin, R. Orava and M. G. Ryskin, Eur. Phys. J. C 25, 391 (2002).

[2] R. Enberg, G. Ingelman, A. Kissavos and N. Tîmneanu, Phys. Rev. Lett. 89, 081801 (2002).

[3] V.A. Khoze, A.D. Martin and M.G. Ryskin, Eur. Phys. J. C 14, 525 (2000); V. A. Khoze, A. D. Martin and M. G. Ryskin, in proc. 8th International Workshop on Deep Inelastic Scattering and QCD (DIS 2000), Liverpool, England, hep-ph/0006005.

[4] V. A. Khoze, A. D. Martin and M. G. Ryskin, "Diffractive Higgs production: Myths and reality," hep$\mathrm{ph} / 0207313$.

[5] A. De Roeck and C. Royon, Acta Phys. Polon. B 33, 3491 (2002).

[6] B. Cox, J. Forshaw and B. Heinemann, Phys. Lett. B 540, 263 (2002).

[7] A. Edin, G. Ingelman and J. Rathsman, Phys. Lett. B 366, 371 (1996); Z. Phys. C75, 57 (1997).

[8] A. Edin, G. Ingelman and J. Rathsman, Phys. Rev. D 56, 7317 (1997); C. Brenner Mariotto, M. B. Gay Ducati and G. Ingelman, Eur. Phys. J. C 23, 527 (2002).

[9] G. Ingelman, A. Edin and J. Rathsman, Comput. Phys. Commun. 101, 108 (1997).

[10] T. Sjöstrand, P. Edén, C. Friberg, L. Lönnblad, G. Miu, S. Mrenna and E. Norrbin, Comput. Phys. Commun. 135, 238 (2001).

[11] B. Andersson, G. Gustafson, G. Ingelman and T. Sjöstrand, Phys. Rept. 97, 31 (1983).

[12] J. Rathsman, Phys. Lett. B 452, 364 (1999).

[13] H.L. Lai et al., Eur. Phys. J. C 12, 375 (2000).

[14] R. Enberg, G. Ingelman and N. Tîmneanu, Phys. Rev. D 64, 114015 (2001).

[15] CDF Collaboration, T. Affolder et al., Phys. Rev. Lett. 85, 4215 (2000).

[16] CDF Collaboration, F. Abe et al., Phys. Rev. Lett. 78, 2698 (1997); CDF Collaboration, F. Abe et al., Phys. Rev. Lett. 79, 2636 (1997); M. Albrow, FERMILABCONF-98-138-E (1998); CDF Collaboration, T. Affolder et al., Phys. Rev. Lett. 84, 232 (2000).

[17] D $\varnothing$ Collaboration, B. Abbott et al., Phys. Lett. B 531, $52(2002)$

[18] M. Grazzini, "Direct Higgs production at hadron colliders," hep-ph/0209302, and references therein.

[19] See e.g., M. Carena et al., "Report of the Tevatron Higgs working group," hep-ph/0010338.

[20] S. J. Brodsky, P. Hoyer, N. Marchal, S. Peigné and F. Sannino, Phys. Rev. D 65, 114025 (2002). 